\title{
Adult T cell leukemia: a tale of two T cells
}

\author{
O. John Semmes
}

Departments of Microbiology and Molecular Cell Biology, and Pathology, Eastern Virginia Medical School, Norfolk, Virginia, USA.

\begin{abstract}
Human T cell leukemia virus type 1 (HTLV-1) is the etiologic agent for the development of an aggressive hematologic neoplasia termed adult $T$ cell leukemia/lymphoma (ATLL). Although the virus infects $\mathrm{T}$ cell subsets that display either CD4 or CD8 cell surface markers, the leukemic cell is exclusively of the $\mathrm{CD4}^{+}$subtype. In the article by Sibon et al. in this issue of the JCI, the authors demonstrate that the molecular basis for clonal expansion differs between these 2 infected $T$ cell populations (see the related article beginning on page 974). The molecular events associated with a preleukemic state, such as genomic instability, polynucleation, and cell cycle redistribution, were only observed in $\mathrm{CD}^{+} \mathrm{T}$ cells. This finding provides a molecular-based mechanism for the restriction of the leukemic phenotype to the $\mathrm{CD}^{+} \mathrm{T}$ cell subtype.
\end{abstract}

\section{HTLV-1 and adult T cell leukemia}

Infection with human $T$ cell leukemia virus type 1 (HTLV-1) leads to the development of an aggressive hematopoietic disease, adult $\mathrm{T}$ cell leukemia/lymphoma (ATLL). The manifestation of ATLL occurs in fewer than $3 \%$ of all cases of infection and follows an asymptomatic period lasting, on average, greater than 30 years. In addition to ATLL, infection can lead to other subneoplastic conditions, most notably HTLV-1associated myelopathy/tropical spastic paraparesis (HAM/TSP), which occurs at approximately the same rate. Infection with HTLV-1 appears to be restricted to T cells expressing CD4 and/or CD8 surface antigens, although secondary involvement of other cell types has been reported $(1,2)$. Although it is clear that HTLV-1 can infect both $\mathrm{CD}^{+}$and $\mathrm{CD} 4^{+} \mathrm{T}$ cells, the ATLL leukemia cell phenotype is restricted to $\mathrm{CD} 4^{+}$ cells. Therefore a major unanswered question in ATLL leukemogenesis is, how does infection of both cell types transform into infection of only one cell type?

One obvious postulate for establishing a $\mathrm{CD}^{+}$restriction would be via receptor-mediated selective advantage in viral entry that favors one cell type. However, the discovery that the $\mathrm{T}$ cell surface protein GLUT-1 serves as a component of the HTLV-1 receptor has provided little information regarding virus entry barriers since

Nonstandard abbreviations used: ATLL, adult T cell leukemia/lymphoma; HAM/TSP, HTLV-1-associated myelopathy/tropical spastic paraparesis; HTLV, human T cell leukemia virus.

Conflict of interest: The author has declared that no conflict of interest exists.

Citation for this article: J. Clin. Invest. 116:858-860 (2006). doi:10.1172/JCI28140.
GLUT-1 is a ubiquitous cell surface protein. Following cell surface contact, various post-entry events are essential regulators of the virus-cell interaction that would impact virus persistence and/or clonal expansion. In addition, specific virus-induced cellular changes resulting from oncogenic molecular events unique to the $\mathrm{CD} 4^{+}$environment might select for $\mathrm{CD} 4^{+}$clones. In this issue of the JCI, Sibon et al. (3) provide strong evidence that although both $\mathrm{CD}^{+}$and $\mathrm{CD}^{+} \mathrm{T}$ cells are infected by HTLV-1, only the $\mathrm{CD}^{+}$infected cells exhibit a preleukemic phenotype. It is particularly interesting that although both infected cell types display clonal expansion and a growth advantage over noninfected cells, the specific molecular pathways toward this end are unique to each cell type.

\section{Restricting a preleukemic phenotype to $\mathrm{CD}^{+} \mathrm{T}$ cells}

Determination of the biological events that occur between virus infection and disease development has been a particularly challenging area of viral pathology research. The specific challenge for HTLV-1 biology is the definition of the preleukemia infected cell. Clearly in the case of ATLL, a preleukemic cell is an HTLV-1-infected cell that is destined to undergo transformation and become the clonal progenitor of ATLL. This cell, of course, cannot be predictably identified prior to transformation. Therefore, Sibon et al. (3) decided to target the next best thing: cells derived from a disease state that is not ATLL. In this study, they isolated cells of specific lineage from individuals suffering from HAM/TSP, using cell surface marker immunoselection. It should be noted here that HTLV-1- infected cells from individuals suffering from HAM/TSP do not exist in a true preneoplastic state and do not become ATLL tumor cells at a higher frequency than do the HTVL-1-infected cells of asymptomatic individuals. However, HTLV-1-infected cells from HAM/TSP patients do represent late-stage infection. This slightly alters the experimental objective from one of defining a known preleukemic cell toward one of examining a postinfection, nonmalignant cell for signs of harboring a potential preleukemic phenotype.

In each of the isolated cell samples, the infected cells were compared with uninfected cells from the same individuals (3). This design allowed for comparison between infected and uninfected cells as well as for observing the differential impact of cell type-specific infection. Indeed, infection of either $\mathrm{CD}^{+}$or $\mathrm{CD}^{+}$cell types resulted in clonal expansion when compared with uninfected cells. Thus HTLV-1 infection confers a growth/survival advantage upon naturally infected $\mathrm{T}$ cells. Although both cell types also showed an increase in DNA synthesis, there were subtle differences in the expansion of the cell types, with $\mathrm{CD}^{+}$ cells showing a greater increase in cell number and $\mathrm{CD} 4^{+}$cells showing a greater number of viral copies. In fact, the most interesting observation may be that the 2 cell types follow separate paths toward clonal expansion. The HTLV-1-infected $\mathrm{CD}^{+}$population showed a resistance to apoptosis that directly correlated with the increased number of cells (Figure 1). However, although HTLV-1-infected $\mathrm{CD} 4^{+}$cells displayed an increase in DNA synthesis, this population did not exhibit an increase in cell numbers. This apparent contradiction was later explained by the demonstration that the infected $\mathrm{CD} 4^{+}$ population displayed a high percentage of multinucleated cells, indicating that the signals for cell division are intact but effective cytokinesis is impaired. The presence of multinucleated cells and the additional observation of chromosomal bridges, both restricted to the $\mathrm{CD} 4^{+}$population, provided clear evidence of loss of genomic integrity; additionally, each is a candidate phenotype for preleukemia (Figure 1). 


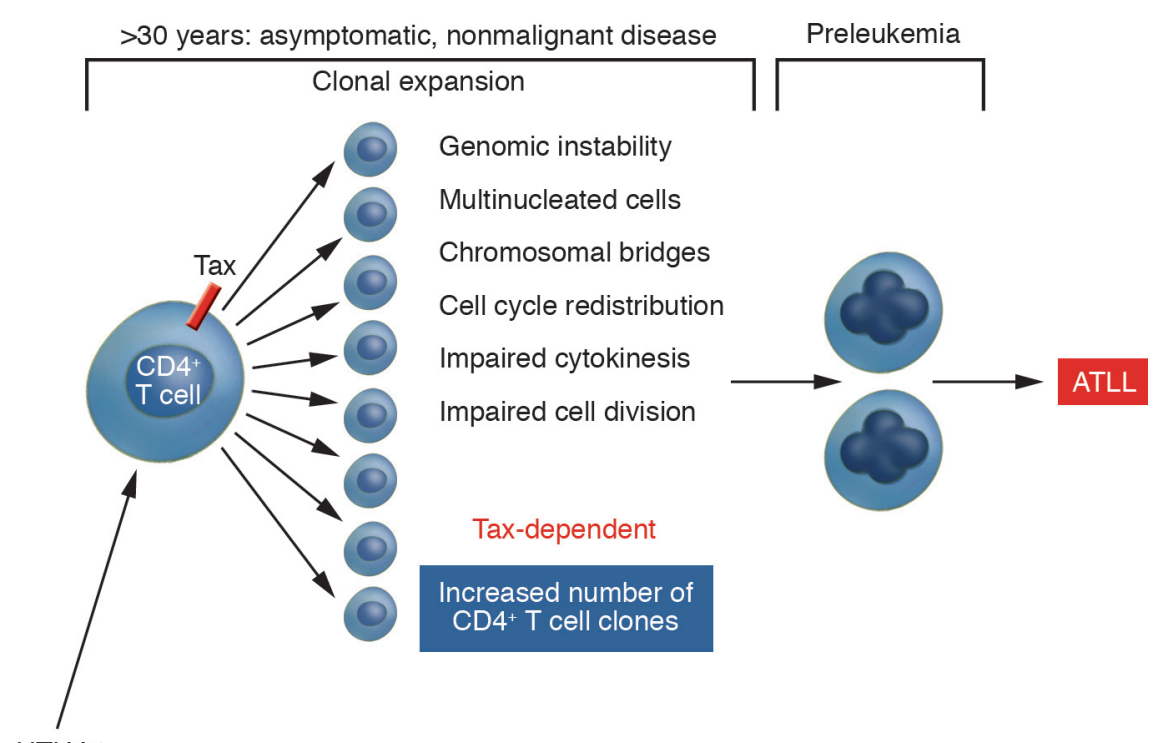

HTLV-1

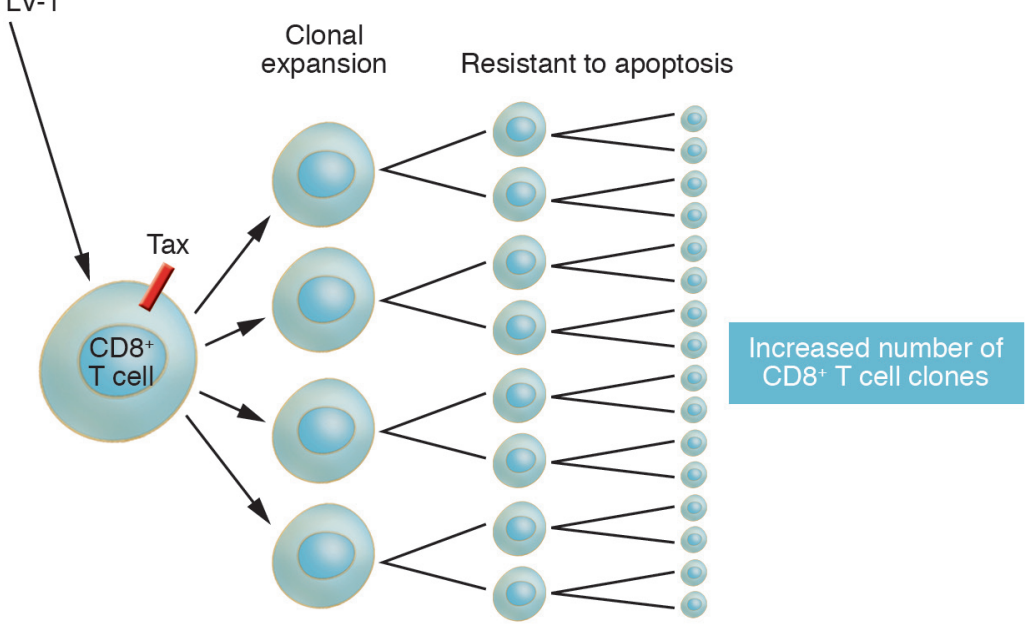

Tax-independent

\section{The role of HTLV-1 Tax protein in the development of ATLL}

It has been postulated by some, and strongly adopted by others, that HTLV-1 infection results in a state of genomic instability that sets the stage for accumulation of the events necessary for leukemogenesis (reviewed in refs. 4, 5). The predicted randomness of such genomic insults is an attractive element of this hypothesis in that it helps explain the long latent period following virus infection prior to disease development. However, if the molecular events are random, then why is there a specific cell type and disease type? In this respect, cell-specific restriction of molecular activities would be a reasonable explanation. The reported oncogenic capabilities of the viral protein Tax revolve around the ability of Tax to alter cell cycle, genomic instability, and apoptosis in a not necessarily unrelated fashion (4, 5). All of these Tax-related activities were confirmed in the ex vivo model presented by Sibon et al. except for apoptosis (3). All in all, the most intriguing consistency may be the result that Taxexpressing cells displayed genetic instability and that de novo expression of Tax resulted in the accumulation of cells in a higher ploidy state $(>2 \mathrm{~N})$. Certainly it is clear that induction of genomic instability, which Sibon et al. show is restricted to $\mathrm{CD}^{+}$cells, is a compelling precondition for cellular transformation in a variety of cancer models. This dichotomy between $\mathrm{CD}^{+}$and $\mathrm{CD}^{+}$cells for accumulation of genomic aberrations, indicative of loss of genomic integrity, is alone sufficient to explain the restriction of ATLL to the $\mathrm{CD}^{+}$cell type and suggests that Tax activities differ between $\mathrm{T}$ cell subtypes.

\section{Figure 1}

HTLV-1 disease and clonal expansion of separate T cell subsets. After HTLV-1 infection, both $\mathrm{CD}^{+}$and $\mathrm{CD} 8^{+} \mathrm{T}$ cell subpopulations exhibit clonal expansion. In this issue of the $\mathrm{JCl}$, Sibon et al. (3) examined each subpopulation using a sophisticated ex vivo model system. The authors observed that although each infected cell type displayed clonal expansion, loss of genomic integrity, which is a necessary predisposition for neoplasia, was observed only in the CD4 $4^{+} \mathrm{T}$ cell population. Clonal expansion of the HTLV-1infected CD8 ${ }^{+} \mathrm{T}$ cell population is associated with increased cell division and resistance to apoptosis. The interaction between the 2 molecular pathways toward clonal expansion utilized by each $T$ cell subset could have significant impact upon which HTLV-1 disease will develop in an infected individual.

As with all molecularly defined systems that utilize cell lines and transient overexpression approaches, arguments abound with respect to which cell system is appropriate and which results are relevant to human disease. In point of fact, although there is universal agreement on the induction of genomic instability, there are contradictory reports with respect to the ability of Tax to induce apoptosis (6-9). Likewise, although most studies support the hypothesis that Tax alters the cell cycle (10-12), some studies fail to make this observation (13). Clearly the results derived from the ex vivo system employed by Sibon et al. (3) demonstrate that Tax expression correlated with the increased percentage of $\mathrm{CD} 4^{+}$cells residing in $\mathrm{S}$ and $\mathrm{G}_{2} \mathrm{M}$ phases but had no such effect in $\mathrm{CD}^{+}$cells. Thus, although each of the molecular systems employed in the transient overexpression studies can shed light on Tax activities, the systems that reproduce genomic instability and altered cell cycle distribution are more biologically relevant to ATLL development.

\section{What's next?}

Obviously the involvement of $\mathrm{CD} 4^{+}$cells in the development of ATLL is indisputable, and uncovering the underlying molecular events in this cell type will have a profound impact on our understanding of this disease. However, the role of HTLV-1-infected $\mathrm{CD}^{+} \mathrm{T}$ cells in disease development is less clear. Is it possible that the infection and expansion of $\mathrm{CD}^{+}$cells plays a helper role in the selection of specific $\mathrm{CD} 4^{+}$cell clones? In fact, what is the dynamic relationship between the two cell populations over the course of disease development? Is there a 
patient-specific variation in the ratios of the infected cell types that might explain why one patient may develop ATLL and another individual develops HAM/TSP? It is predictable that future work will seek to uncover the dynamics of multiple virusinfected cell populations and what impact these forces have on the selection and manifestation of HTLV-1-related diseases.

Address correspondence to: O. John Semmes, Departments of Microbiology and Molecular Cell Biology and Pathology, Eastern Virginia Medical School, Norfolk, Virginia 23501, USA. Phone: (757) 446-5904; Fax: (757) 4465766; E-mail: semmesoj@evms.edu.

1. Hirayama, M., et al. 1988. Infection of human T-lymphotropic virus type I to astrocytes in vitro with induction of the class II major histocompat- ibility complex. Neurosci. Lett. 92:34-39.

2. Hoffman, P.M., et al. 1992. Human T-cell leukemia virus type I infection of monocytes and microglial cells in primary human cultures. Proc. Natl. Acad. Sci.U. S. A. 89:11784-11788.

3. Sibon, D., et al. 2006. HTLV-1 propels untransformed $\mathrm{CD}^{+}$lymphocytes into the cell cycle while protecting $\mathrm{CD}^{+}$cells from death. J. Clin. Invest. 116:974-983. doi:10.1172/JCI27198.

4. Jeang, K.T., Giam, C.Z., Majone, F., and Aboud, M. 2004. Life, death, and tax: role of HTLV-I oncoprotein in genetic instability and cellular transformation. J. Biol. Chem. 279:31991-31994.

5. Marriott, S.J., and Semmes, O.J. 2005. Impact of HTLV-I Tax on cell cycle progression and the cellular DNA damage repair response. Oncogene. 24:5986-5995.

6. Chlichlia, K., et al. 1997. ICE-proteases mediate HTLV-I Tax-induced apoptotic T-cell death. Oncogene. 14:2265-2272.

7. Chlichlia, K., et al. 1995. Immediate effects of reversible HTLV-1 tax function: T-cell activation and apoptosis. Oncogene. 10:269-277.

8. Copeland, K.F., Haaksma, A.G., Goudsmit, J., Krammer, P.H., and Heeney, J.L. 1994. Inhibition of apoptosis in T cells expressing human $\mathrm{T}$ cell leukemia virus type I Tax. AIDS Res. Hum. Retroviruses. 10:1259-1268.

9. Yamada, T., et al. 1994. The human T-cell leukemia virus type I Tax protein induces apoptosis which is blocked by the Bcl-2 protein. J. Virol. 68:3374-3379.

10. Haoudi, A., Daniels, R.C., Wong, E., Kupfer, G., and Semmes, O.J. 2003. Human T-cell leukemia virus-I tax oncoprotein functionally targets a subnuclear complex involved in cellular DNA damageresponse. J. Biol. Chem. 278:37736-37744.

11. Lemoine, F.J., and Marriott, S.J. 2001. Accelerated $\mathrm{G}(1)$ phase progression induced by the human $\mathrm{T}$ cell leukemia virus type I (HTLV-I) Tax oncoprotein. J. Biol. Chem. 276:31851-31857.

12. Liang, M.H., Geisbert, T., Yao, Y., Hinrichs, S.H., and Giam, C.Z. 2002. Human T-lymphotropic virus type 1 oncoprotein tax promotes S-phase entry but blocks mitosis. J. Virol. 76:4022-4033.

13. Park, H.U., Jeong, S.J., Jeong, J.H., Chung, J.H., and Brady, J.N. 2005. Human T-cell leukemia virus type 1 Tax attenuates gamma-irradiation-induced apoptosis through physical interaction with Chk2. Oncogene. 25:438-447.

\title{
Oxidative stress in sepsis: a redox redux
}

\author{
Jay K. Kolls \\ Children's Hospital of Pittsburgh, Pittsburgh, Pennsylvania, USA.
}

\begin{abstract}
Sepsis and sepsis syndrome are leading causes of mortality throughout the world. It is widely held that sepsis represents a dysregulated innate immune response to an offending pathogen. This immune response is often initiated via microbial products signaling through TLRs expressed on host immune cells. There is increasing evidence that this innate response can be dramatically influenced by the cellular redox state, and thus a better understanding of oxidative regulation of innate immunity could lead to new treatments for sepsis. In this issue of the JCI, Thimmulappa et al. show that nuclear factorerythroid 2-related factor 2 (Nrf2), a member of the "cap'n'collar" family of basic region-leucine zipper transcription factors, which has previously been shown to be involved in the transcription of antioxidant gene expression in response to xenobiotic stress, is also a critical regulator of cellular oxidative stress in sepsis (see the related article beginning on page 984).
\end{abstract}

Despite decades of advances in antibiotic treatment, sepsis remains an elusive killer, with over 750,000 cases per year in North America (1) with a $40-50 \%$ mortality rate in adults. Sepsis is mediated by infectious stimuli, and many of the clinical findings of sepsis can be replicated in experimental animal models using specific bacterial

Nonstandard abbreviations used: IKK, inhibitor of $\kappa B$ kinase; IRAK, IL-1 receptor-associated kinase; IRF-3, IFN regulatory factor 3; NAC, $\mathrm{N}$-acetyl cysteine; Nrf2, nuclear factor-erythroid 2-related factor 2; TIR, Toll/IL-1 receptor; TRIF, TIR domain-containing adaptor inducing IFN- $\beta$.

Conflict of interest: The author has declared that no conflict of interest exists.

Citation for this article: J. Clin. Invest. 116:860-863 (2006). doi:10.1172/JCI28111. products such as LPS (2). The last decade of immunological research has revolutionized how scientists understand the initiation of the innate immune response to invading pathogens. For many offending agents, the TLR family of proteins functions as the host sentinel to invading pathogens. This was first demonstrated in Drosophila melanogaster in 1996 (3), where Toll was shown to regulate the production of the antifungal molecule dorsomycin, and later in mammals, when positional cloning revealed Tlr4 to be the Lps gene product (4). An additional 10 human TLRs have been described that recognize other bacterial products as well as fungi and viruses. These receptors signal via their Toll/IL-1 receptor (TIR) domains using 4 adaptor proteins: (a) MyD88; (b)
TIR domain-containing adaptor inducing IFN- $\beta$ (TRIF); (c) MyD88 adaptor-like/ TIR-associated protein (MAL/TIRAP); and (d) TRIF-related adaptor molecule (TRAM) (2). In the case of LPS signaling through TLR4, the MyD88-dependent pathway is critical for NF- $\mathrm{KB}$ activation and the production of TNF- $\alpha$ whereas the MyD88independent, TRIF-dependent pathway is required for type I IFN production. Based on the fact that 10 human TLRs signal via 4 adaptors and 2 predominant kinases to subsequently regulate the expression of hundreds of genes, the innate immune response has been proposed to be shaped like an hourglass (Figure 1). The top of the hourglass is wide, indicating that 10 TLR proteins recognize a variety of potential offending pathogens, then the hourglass narrows to represent a smaller number of highly conserved TLR adaptor proteins and initial kinases, and then it widens again to reflect the increased number of genes that are transcriptionally activated by NF- $\mathrm{KB}$ and other transcription factors (2). This notion is further supported by the fact that this signaling pathway is markedly conserved among mammalian species, and mutations in this pathway in humans that lead to defective TLR signaling are associated with the development of invasive meningococcal (5) or Legionella 